\title{
Analysis of a narrowband terahertz signal generated by a unitravelling carrier photodiode coupled with a dual-mode semiconductor Fabry-Pérot laser
}

\author{
Sylwester Latkowski, ${ }^{1}$ Josué Parra-Cetina, ${ }^{1}$ Ramón Maldonado-Basilio, ${ }^{1}$ \\ Pascal Landais, ${ }^{1, a)}$ Guillaume Ducournau, ${ }^{2}$ Alexandre Beck, ${ }^{2}$ Emilien Peytavit, ${ }^{2}$ \\ Tahsin Akalin, ${ }^{2}$ and Jean-François Lampin ${ }^{2}$ \\ ${ }^{1}$ Research Institute for Networks and Communications Engineering, Dublin City University, \\ Glasnevin, Dublin 9, Ireland \\ ${ }^{2}$ Institut d'Electronique de Microélectronique et de Nanotechnologie (IEMN), UMR CNRS 8520 Université \\ de Lille 1, Avenue Poincaré B.P. 60069, 59652 Villeneuve d'Ascq, France
}

(Received 8 April 2010; accepted 17 May 2010; published online 15 June 2010)

\begin{abstract}
A narrowband terahertz signal generated by a unitravelling carrier photodiode (UTC-PD) interfaced with a dual-mode Fabry-Pérot laser diode is demonstrated. A beat tone corresponding to the free spectral range is generated on the UTC-PD, and radiated by a transverse-electromagnetic-horn antenna. A terahertz signal at a frequency of $372 \mathrm{GHz}$, featuring a linewidth of $17 \mathrm{MHz}$ is recorded by a subharmonic mixer coupled to an electrical spectrum analyzer. All components involved in this experiment operate at room temperature. The linewidth and the frequency of the emitted terahertz wave are analyzed, along with their dependency on dc-bias conditions applied to laser diode. (C) 2010 American Institute of Physics. [doi:10.1063/1.3447931]
\end{abstract}

Quite recently, it has been demonstrated that semiconductor lasers can produce a terahertz $(\mathrm{THz})$ emission by either using direct ${ }^{1,2}$ or indirect ${ }^{3,4}$ schemes. In the direct generation scheme, the semiconductor lasers used are dc-biased and operate at room temperature $\left(\sim 25{ }^{\circ} \mathrm{C}\right)$. They feature a one-dimensional photonic band gap, introduced by shallow grooves etched on the p-side of the laser junction, perpendicular to the longitudinal axis of the device. These grooves affect the effective refractive index experienced by the electromagnetic wave generated in the active layer. Placement of the grooves at key positions, allow engineering of variations in the refractive index along the laser cavity, and consequently control of the longitudinal spectrum. The FabryPérot (FP) resonance determines an initial spectral separation between consecutive modes but the etched band gap suppresses or enhances some specific FP modes. It is feasible therefore to select modes in order to achieve a given freespectral range. In this emission scheme, an initial design has proven $\mathrm{THz}$ emission at $370 \mathrm{GHz}$, and with the following, generation of a $1.1 \mathrm{THz}$ signal has been indirectly demonstrated. ${ }^{1,5}$ The origin of the $\mathrm{THz}$ generation in this context is attributed to the third order intraband nonlinear effects inside laser cavity. ${ }^{5}$

The demonstration of indirect generation, was first performed with dual-mode microchip laser ${ }^{3}$ at $101.5 \mathrm{GHz}$, and subsequently with other structures including distributed Bragg reflector laser diode ${ }^{4}$ at $163.5 \mathrm{GHz}$. They were used to produce an intermediate beat frequency on a lowtemperature grown GaAs photodetector. ${ }^{6}$

The linewidth of the $\mathrm{THz}$ emission has not been analyzed, independently of the generation scheme used, due to the limitations of the resolution of detection setups. The main objective of this paper is to experimentally investigate the linewidth of the THz signal generated by an indirect technique featuring a slotted FP laser diode and a unitravelling

${ }^{a)}$ Electronic mail: landaisp@eeng.dcu.ie. carrier photodiode (UTC-PD). The THz linewidth should be in order of the summary linewidth of the optical modes contributing to the generation of such a signal, although it could be further reduced as a result of high coherence of the optical modes originating from a single laser cavity. Our experimental scheme is presented in Fig. 1. Two key components are used to generate a $\mathrm{THz}$ signal: a spectrum tailored semiconductor FP laser denoted as a device under test (DUT), and a UTC-PD producing the photomixing at $\mathrm{THz}$ frequencies. The detection and the analysis of the $\mathrm{THz}$ signal are achieved by a high bandwidth electrical spectrum analyzer (ESA). Along with these components, standard elements for optical tests and measurement techniques are used. The entire setup and its components are placed on an antivibrational optical workbench, and the measurements are carried out at room temperature $\left(\sim 25^{\circ} \mathrm{C}\right)$. DUT is mounted in a standard T0 can. It is a $350 \mu \mathrm{m}$ long, InAlGaAs multiquantum well based FP laser as previously described in Refs. 1 and 7. Its $2 \mu \mathrm{m}$ wide ridge waveguide secures a single mode spatial output. The grooves are $1 \mu \mathrm{m}$ deep and span across the ridge. It is characterized by a threshold current of $I_{\mathrm{th}}=19 \mathrm{~mA}$. Two longitudinal modes located at 1553

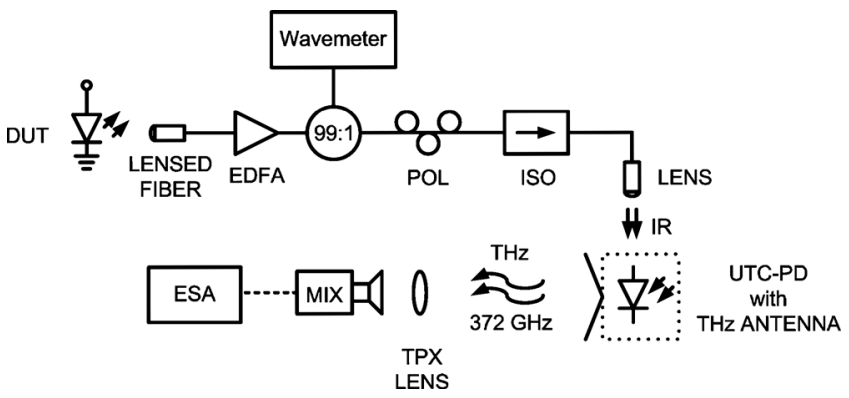

FIG. 1. Experimental setup for the generation and characterization of $\mathrm{THz}$ signal. All fiberised and free space components operate at room temperature. Optical fiber is represented with a solid line and electrical path is represented with dashed line. 


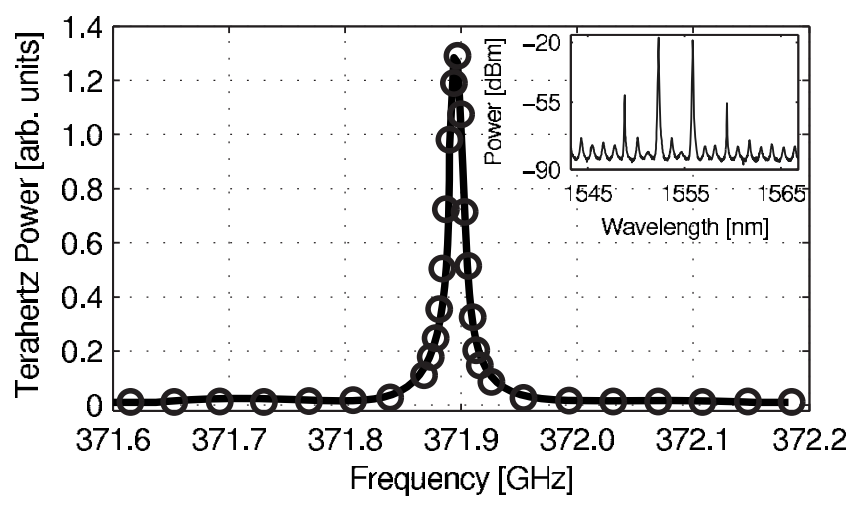

FIG. 2. THz spectrum recorded for the DUT biased at $88.5 \mathrm{~mA}$ and temperature controlled at $25^{\circ} \mathrm{C}$. Measured data are represented by $(\bigcirc)$, the fitted Lorentzian distribution is represented by a solid line. In inset the optical spectrum recorded with a resolution of $0.02 \mathrm{~nm}$, for the same bias and temperature conditions of the DUT.

and $1556 \mathrm{~nm}$, dominate its emission spectrum. They may be accompanied with lateral modes resulting from four-wavemixing (FWM), indicating strong nonlinear interactions of optical fields inside the laser cavity. This depends on the bias conditions. However, the optical power unbalance (PU) between the two main modes and the side-bands is greater than $30 \mathrm{~dB}$, suggesting that their direct contribution to the beat signal is negligible. The optical linewidths of the two main modes are in the order of several $\mathrm{MHz}^{7}$, which should lead to a THz linewidth of the same order. ${ }^{4}$ The UTC-PD is monolithically integrated with a transverse-electromagnetic-horn antenna (TEM-HA) (Refs. 8 and 9) for optimum coupling between the photodetector and the free space. THz generation has been demonstrated up to $1800 \mathrm{GHz}$ with this device. $^{10}$

The infrared output of the DUT is collected by a lensed optical fiber, with $50 \mu \mathrm{W}$ of average power coupled. An erbium doped fiber based optical amplifier (EDFA) provides a constant optical output power, set at $13 \mathrm{dBm}$, the level required for UTC-PD to operate efficiently without bias current provided, after consideration of losses introduced by other elements of the setup. A wavemeter allows continuous monitoring of the spectral output from the DUT. The polarization controller is used to adjust the polarization state of the infrared signal directed onto the UTC-PD. The optical isolator prevents any spurious feedback provided by reflections from the surface of the photodetector. A THz signal is generated on the UTC-PD and propagated via TEM-HA. A polymethylpentene (TPX) based lens, with focal length of $25 \mathrm{~mm}$ is used, in order to collect and focus the $\mathrm{THz}$ radiation, into a WR-2.8 (260-400 GHz) rectangular metallic waveguide of an active subharmonic mixer (MIX). This mixer is driven by $7-15.5 \mathrm{GHz}$ local oscillator followed by an active multiplier. The intermediate frequency signal at 404.4 MHz is resolved with an ESA, with a resolution bandwidth of $1 \mathrm{MHz}$ and a sweep time of $20 \mathrm{~ms}$, with no averaging. Figure 2 shows a record of the $\mathrm{THz}$ signal generated with this setup. The DUT is biased at $88.5 \mathrm{~mA}$ and temperature controlled at $25^{\circ} \mathrm{C}$ and no noticeable central frequency fluctuations were observed in a few minutes timescale. In the inset, under the same operating conditions, the optical spectrum recorded at the laser output is presented. It is possible to generate a $\mathrm{THz}$ signal using the indirect scheme, based on the multimode semiconductor laser and the UTC-PD photo-

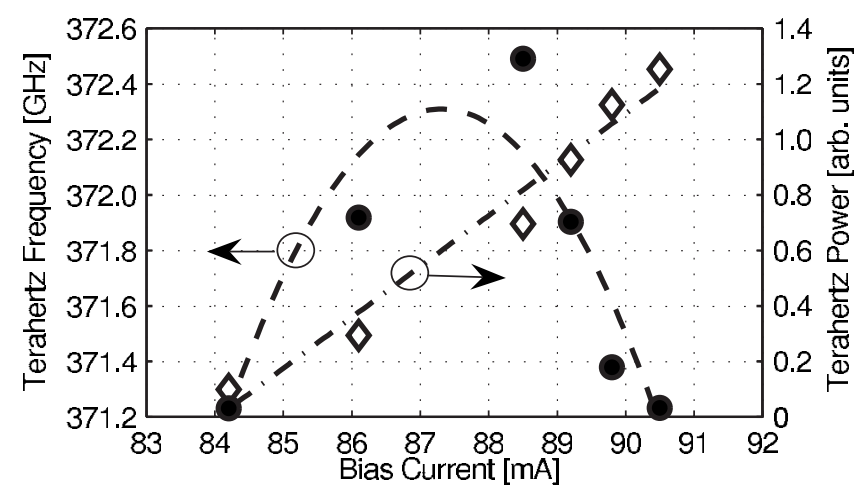

FIG. 3. Power of THz signal generated $(\bullet)$ and its central frequency $(\diamond)$ as a function of bias current applied to the laser diode. The dashed lines are eye-guides.

detector. The $\mathrm{THz}$ signal can be fitted with a Lorentzian shape, exhibiting a central frequency at $371.895 \mathrm{GHz}$, and a linewidth of $(16.89 \pm 0.12) \mathrm{MHz}$. This frequency corresponds to the free spectral range between the main optical modes as depicted in the inset of Fig. 2. It is possible to achieve narrower $\mathrm{THz}$ linewidth by using two single mode external cavity lasers (ECL) as the optical mode sources, which are characterized by the optical linewidths in the order of $100 \mathrm{kHz}$. However, such a scheme is prone to the central frequency fluctuations which arise from thermal and mechanical instabilities. An active phase-locking setup allowing to overcome these effects, ${ }^{11}$ would mostly confine it to a laboratory environment. The impact of such instabilities, if present, in the case of a monolithic dual-mode laser diode is negligible, as both modes simultaneously experience the same fluctuations. Therefore with our device, the central frequency of the $\mathrm{THz}$ wave exhibits virtually no shift when recorded at given experimental conditions, as opposed to the one achieved with two ECL sources where the measured values are sweep time dependant due to such instabilities. Furthermore, the linewidth of the $\mathrm{THz}$ signal can benefit from the coherence of the optical modes emitted by our laser diode, enhanced via intracavity $\mathrm{FWM}^{7}{ }^{7}$ which is not the case with two independent single mode sources, as their phases are uncorrelated. Such a $\mathrm{THz}$ spectrum has been recorded as a function of the bias current applied to the DUT, while its temperature is maintained at $25^{\circ} \mathrm{C}$. Figure 3 compiles the central frequency of the recorded $\mathrm{THz}$ signal and the estimated power associated with $\mathrm{THz}$ peak for a range of bias between 84 and $91 \mathrm{~mA}$. Outside this current range, it is not possible to detect and to resolve the $\mathrm{THz}$ signal. The power is expressed in arbitrary unit as it was difficult to evaluate the loss between the free space $\mathrm{THz}$ emission and the waveguide coupling. A linear dependence with positive slope of central frequency is clearly observed, across a $1200 \mathrm{MHz}$ tuning range. This behavior can be linked to the variation in the group refractive index of the active layer. As the bias current increases, the carrier density in the active layer increases. By plasma effect the group refractive index is reduced. The free spectral range increases and therefore the beat frequency also increase. The $\mathrm{THz}$ signal power increases with bias current, until it reaches a maximal value for a bias of $89 \mathrm{~mA}$. The power decreases with further increase in current. If we keep in mind that the optical power launched into the UTC-PD is constant, this $\mathrm{THz}$ power variation is due a variation in the spectrum of the dual-mode laser. Furthermore, the linewidth 


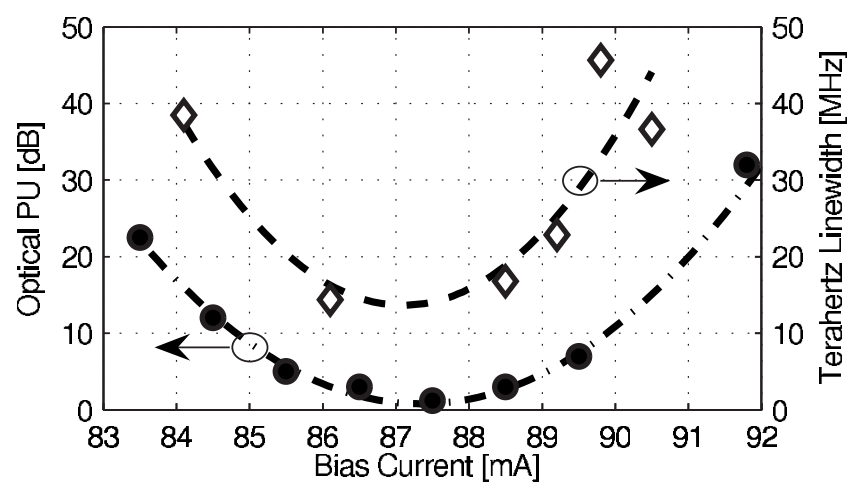

FIG. 4. Optical power unbalance $(\diamond)$ and $\mathrm{THz}$ linewidth $(\diamond)$ as a function of bias current applied to the laser diode. The dashed lines are eye-guides.

of the $\mathrm{THz}$ signal as function of the bias current and the PU between the two dominant optical modes for the same bias range are plotted in Fig. 4. Outside the range of bias current between 84 and $91 \mathrm{~mA}$, where the THz emission occurs, the PU is larger than $20 \mathrm{~dB}$. At $83.5 \mathrm{~mA}$ the dominant mode is at $1553 \mathrm{~nm}$ while at $92 \mathrm{~mA}$, it is at $1556 \mathrm{~nm}$. The spectral purity of the $\mathrm{THz}$ signal appears to be linked to the PU. In the investigated bias range both quantities seem to follow a similar trend, achieving minimal values at the same bias current. This can be understood by the fact that in these optical spectral conditions, the coherence of the optical modes is enhanced. The intracavity FWM contributes to reduce the phase noise of each mode, and therefore their linewidth.

In this paper, we demonstrated the generation of a narrowband $\mathrm{THz}$ signal based on a spectrum tailored semiconductor FP laser and a UTC-PD, at room temperature. The key feature of this scheme lies in the stability and the quality of the THz signal achieved. The THz wave generated is measured at $371.9 \mathrm{GHz}$, with a tunability of $1200 \mathrm{MHz}$ for a variation in the bias current in a range from 84 to $91 \mathrm{~mA}$. Its linewidth is measured at $17 \mathrm{MHz}$ which corresponds to the order of the linewidths associated with the optical modes. Results of the order of $10 \mathrm{kHz}$ have been achieved with glass lasers, ${ }^{12}$ however they are complex, need centimeter-long cavity with precise and stable mechanics, and offer a lower efficiency due to optical pumping. For applications compatible with megahertz linewidths semiconductor lasers are more compact and cost effective. It should be noted that if the optical coupling between the laser and the fiber is enhanced the EDFA could be removed from the setup. In this case a very compact, light-weight, and possibly battery operated $\mathrm{THz}$ source can be achieved.

The authors wish to acknowledge HEA PRTLI4 INSPIRE, CNRS, and the "Région Nord-Pas-de-Calais" for their support. We thank X. Wallart, C. Coinon, and F. Mollot for the epitaxial growth of the UTC-PD, and M. Zaknoune and D. Ducatteau for fruitful discussions about the process and the $\mathrm{THz}$ spectrum analysis.

${ }^{1}$ S. Latkowski, F. Surre, and P. Landais, Appl. Phys. Lett. 92, 081109 (2008).

${ }^{2}$ S. Hoffmann, M. R. Hofmann, M. Osinski, H. Amano, and F. Henneberger, Physics and Simulation of Optoelectronic Devices XII (SPIE, San Jose, CA, 2004), Vol. 5349, pp. 218-227.

${ }^{3}$ M. Hyodo, M. Tani, S. Matsuura, N. Onodera, and K. Sakai, Electron. Lett. 32, 1589 (1996)

${ }^{4}$ M. Tani, P. Gu, M. Hyodo, K. Sakai, and T. Hidaka, Opt. Quantum Electron. 32, 503 (2000).

${ }^{5}$ S. Latkowski, F. Surre, R. Maldonado-Basilio, and P. Landais, Appl. Phys. Lett. 93, 241110 (2008).

${ }^{6}$ E. Peytavit, G. Mouret, J.-F. Lampin, S. Arscott, P. Masselin, L. Desplanque, O. Vanbesien, R. Bocquet, F. Mollot, and D. Lippens, IEE Proc.: Optoelectron. 149, 82 (2002).

${ }^{7}$ R. Maldonado-Basilio, S. Latkowski, F. Surre, and P. Landais, Opt. Commun. 283, 299 (2010).

${ }^{8}$ E. Peytavit, J.-F. Lampin, T. Akalin, and L. Desplanque, Electron. Lett. 43, 73 (2007).

${ }^{9}$ A. Beck, M. Zaknoune, E. Peytavit, T. Akalin, G. Ducournau, J.-F. Lampin, F. Mollot, F. Hindle, C. Yang, and G. Mouret, Proceedings of the 33rd International Conference on Infrared, Millimeter, and Terahertz Waves (IRMMW-THz), Pasadena, CA, USA, 2008, pp. 1-2.

${ }^{10}$ A. Beck, G. Ducournau, M. Zaknoune, E. Peytavit, T. Akalin, J.-F. Lampin, F. Mollot, F. Hindle, C. Yang, and G. Mouret, Electron. Lett. 44, 1320 (2008).

${ }^{11}$ Q. Quraishi, M. Griebel, T. Kleine-Ostmann, and R. Bratschitsch, Opt. Lett. 30, 3231 (2005).

${ }^{12}$ M. Alouini, M. Brunel, F. Bretenaker, M. Vallet, and A. Le Floch, IEEE Photonics Technol. Lett. 10, 1554 (1998). 